\title{
Length of hospital stay in treatment of venous thromboembolism: do outcomes vary according to preference of anticoagulant? A retrospective analysis
}

\author{
Iftikhar Haider Naqvi ${ }^{1^{*}}$, Abu Talib², Rizvi Zehra Saiyeda Nayema ${ }^{1}$, Muhammad Syed ${ }^{3}$, \\ Rija Ghazanfar ${ }^{4}$, Syeda Kashaf Fatima ${ }^{5}$ \\ ${ }^{1}$ Lifeline Medical Center, Karachi, Pakistan \\ ${ }^{2}$ Burhani Hospital, Karachi, Pakistan \\ ${ }^{3}$ Alkidhmat Hospital, Karachi, Pakistan \\ ${ }^{4} \mathrm{~K}$-Health Care Hospital, Karachi, Pakistan \\ ${ }^{5}$ Move Diabetic and Medical Center, Karachi, Pakistan
}

\begin{abstract}
Introduction: Regarding the choice of novel or traditional oral anticoagulants for the treatment of different entities of venous thromboembolism (VTE), there is contrasting, little, or no evidence to put forward. We here assess the impact of various anticoagulants in reducing the length of stay (LOS) in patients with acute VTE. Our objectives were: 1) to compare LOS among novel and traditional anticoagulants groups on discharge, and 2) to determine the clinical risk factors responsible for lengthier hospital stay in patients having acute VTE.

Material and methods: We conducted a retrospective data analysis of 161 consecutively admitted patients in the Life line Hospital, Karachi, Pakistan with a recent diagnosis of VTE. Lengths of stay with various anticoagulants on discharge were compared. Bleeding complications and readmission outcomes were compared, along with determination of independent predictors by multivariate analysis for LOS among groups.

Results: Patients discharged on a vitamin $\mathrm{K}$ antagonist (warfarin) had significantly longer hospital stays compared to patients on rivaroxaban (7.65 days vs. 5.21 days, $p<0.001$ ). Patients discharged exclusively on enoxaparin (hospital stay duration of 3.30 days) or on a combination of enoxaparin and warfarin (hospital stay duration of 4.26 days,) when compared for LOS for rivaroxaban, showed statistical significance $(p<0.0001)$.

Conclusions: Warfarin has significantly longer LOS compared to rivaroxaban. Bleeding outcomes and readmissions compared among anticoagulant discharged groups were found to be statistically insignificant. Novel anticoagulants have an observable impact on the length of hospital stay in patients with acute venous thromboembolism.
\end{abstract}

Key words: venous thromboembolism, pulmonary embolism, rivaroxaban, enoxaparin, warfarin

Acta Haematologica Polonica 2021; 52, 6: 590-596

\footnotetext{
*Address for correspondence: Iftikhar Haider Naqvi,

Lifeline Medical Center, Karachi, 74200 Pakistan,

phone +92 3003655958 ,

e-mail: drihnaqvi@gmail.com
}

PTHiT

Received: 09.12.2020

Accepted: 31.12.2020

Copyright (C) 2021

The Polish Society of Haematologists and Transfusiologists, Insitute of Haematology and Transfusion Medicine.

All rights reserved. 


\section{Introduction}

Acute venous thromboembolism has long been a grave medical challenge worldwide, and is evidently associated with multiple medical disorders and situations. Deep vein thrombosis (DVT), and pulmonary embolism (PE), either or both, are the typical manifestations of acute venous thromboembolism (VTE), where venous thromboembolism is a frequently encountered ailment, having a yearly incidence of 1 to 2 per 1,000 people [1, 2]. The symptomatology ranges from swelling of an extremity, shortness of breath, to shock or death. Its management depends on the position along with the degree of the VTE, wherein anticoagulation therapy, systemic thrombolysis or thrombolysis via catheterization, and surgical embolectomy, are the most widely used methods. The gold standard in any form of venous thromboembolism is the institution of anticoagulation. The initiation of therapy is with any parenteral agent along with the later introduction of other more novel agents e.g. oral anticoagulants dabigatran, rivaroxaban, or apixaban in a controlled manner under vigilant observation. These agents are far more convenient to use as the need for spanning or tapering has almost been abolished with their introduction to the anticoagulant profile. Although anticoagulants have added positively to the venous thromboembolism armamentarium, there are health and financial challenges. In the USA alone, around 600,000 cases of VTE are admitted yearly and despite appropriate treatment, mortality remains high $[3,4]$.

Where the early therapy involves parenteral anticoagulation, the goal is to prevent extension and recurrence of any sort of thrombosis [5]. Deep venous thrombosis, although acute, is treated mostly at home rather than in hospital, while pulmonary embolism is an acute medical emergency which is essentially treated in hospital keeping in mind its higher short-term mortality [5]. Conventionally, subcutaneous or intravenous heparin, either low-molecular-weight heparin or unfractionated heparin, is instituted initially combined with a vitamin $\mathrm{K}$ antagonist (VKA), often warfarin, given alongside or immediately after the heparin has been initiated [5]. There are many restrictions on the use of VKAs, such as slow-acting warfarin having a narrow therapeutic range and volatile anticoagulation because of various interactions and inconstant metabolism [6]. So, in order to acquire the desired anticoagulation effects, constant monitoring and accurate dose adjustment are mandatory.

As far as the domain of anticoagulants is concerned, there are some more novel and promising agents being offered in the USA, such as dabigatran (a direct thrombin inhibitor), rivaroxaban, edoxaban, and apixaban (factor Xa inhibitors). When compared to the traditional anticoagulants, the newer agents demonstrate swifter inception with few adverse effects and interactions, and a hassle-free follow up investigative routine [6].
As far as management and monitoring in acute venous thromboembolic states such as DVT and PE are concerned, both the EINSTEIN-DVT and EINSTEIN-PE trials have found rivaroxaban to be equally as effective, if not more so, as enoxaparin and warfarin [7-9]. The same EINSTEIN trials, using the inpatient records of admissions retrospectively, revealed a substantially reduced hospital stay in those patients who received anticoagulation through rivaroxaban compared to those who received enoxaparin/VKA [10]. Another Canadian/USA analysis of EINSTEIN-DVT and EINSTEIN-PE patients also established a mean reduction of about 1.6 days in hospital stay for rivaroxaban-treated patients compared to others treated with enoxaparin/VKA [11].

The shorter length of stay, and reduced readmissions, for treatment with rivaroxaban as compared to warfarin and other contemporary agents also has the benefit of being more cost effective. This has been validated by a case-control study over a period of six months [12].

The available data, apart from clinical studies, is scanty to serve the purpose of validating rivaroxaban as a superior anticoagulant. Having seen the evidence of earlier studies from other parts of the world, in an effort to validate the results, we carried out this study aiming to demonstrate the same superiority of rivaroxaban over other anticoagulants in shortening LOS in hospital, improving hassle-free management and monitoring of anticoagulation, as well as reducing the number of readmissions.

\section{Materials and methods}

This was a retrospective data analysis of patients consecutively admitted to the Life line Hospital in Karachi, Pakistan with a recent diagnosis of VTE between January and December 2019. All the patients, who had a confirmed diagnosis of VTE, including DVT and PE, were retrieved from hospital records. 161 patients in total having VTE were enrolled for the study duration. Patients aged over 18 years who had a confirmed diagnosis of VTE were included. Patients with evidence of VTE within 24 hours of admission, nosocomial infections, iatrogenic overinfusion, pregnancy, or who had any contraindication to anticoagulation were excluded. Patients with a high risk of PE as evidenced by surgical embolectomy or catheter-delivered systemic thrombolytic or thrombolytic agents were also excluded from the study.

\section{Deep vein thrombosis}

The criteria from the American College of Radiology were applied in order to confirm the diagnosis through sonology in accordance with the criteria entailing venous non-compressibility of the involved vein with thrombus echogenicity from within the venous lumen, venous distension of vein, complete Doppler or spectral signal loss from within venous lumen, absent flow phaticity, and muted Valsalva or augmentation response [13]. 


\section{Pulmonary embolism}

Patients suspected for pulmonary embolism were stratified by using a modified Wells Score [14]: $>4$ for a likely diagnosis, $<4$ for unlikely, along with $D$ dimer level. Patients with a modified Wells Score $>4$ were further confirmed objectively via a computerized tomography pulmonary angiogram (CTPA). Pulmonary embolism was confirmed by the following criteria $[15,16]$ on CTPA:

- A large filling defect due to arterial occlusion and enlarged size with respect to adjacent vessels;

- A 'polo mint' sign (a partial filling defect enclosed by contrast material) evidenced on images attained vertical to the long axis of a vessel and a 'railway track' sign (demonstrated on vertical images of the vessel);

- Acute angles formed with the arterial wall due to a peripheral intraluminal filling defect.

\section{Primary and secondary outcomes}

The primary outcome of our study was length of stay in hospital. Bleeding complications, either during index admission or on subsequent readmission, were considered as the secondary outcome.

\section{Bleeding complications}

Bleeding complications were defined and classified in accordance with a previously published study [17] where patients had central nervous system bleed, a fall of hemoglobin level to $>2 \mathrm{~g} / \mathrm{dL}$, and the prerequisite transfusion of two or more packed cell units. Patients who did not meet the major bleeding criteria were labeled as having minor bleeding complications.

\section{Statistical analysis}

Demographic features and clinical physiognomies of the patients on different anticoagulants were shown with their frequency, means \pm standard deviation or median (inter-quartile range). For comparison among the groups, one-way analysis of variance (ANOVA), Kruskal-Wallis test and exact Pearson's Chi-square test were applied as required. Statistical association between anticoagulants and length of stay in hospital among the patients having VTE were determined by Kruskal-Wallis test. For multiple comparison of length of hospital stay within four types of anticoagulant discharge medication in patients having VTE, Bonferroni's adjustment was applied. A Wilcoxon test was used for variables (categorical) having two categories. For multiple categories of categorical variables among groups, we used a Kruskal-Wallis test.

\section{Results}

In our study, multiple demographic and clinical risk factors were taken into account and statistically analyzed for prolonged hospital stay or outcome with treatment of VTE with various anticoagulation agents.

\section{Demographic parameters}

Among demographic profiles, the mean ages of patients on rivaroxaban, on warfarin, on both enoxaparin and warfarin, and on enoxaparin alone, were $60.02 \pm 10.4$, $55.50 \pm 14.68,59.69 \pm 11.54$, and $56.37 \pm 13.46$ years respectively. Of the 161 patients, there were 71 (44.09\%) males and 90 (55.90\%) females in all groups. Gender distribution and ages among various groups are set out in Table I.

\section{Clinical risk factors}

Nearly half of the patients, 69/161 (42.8\%), were discharged on warfarin, where patients with DVT and DM were in the majority. 49/161 (30.43\%) patients were discharged on rivaroxaban as an anticoagulant, where the majority of patients had DVT, DM and malignancies. 30 patients (18.6\%) were discharged on combined enoxaparin and warfarin, with the majority having DM and DVT. Various clinical risk factors such as gender, age, hyperthrombophilic state, malignancies, hormones, immobilization, chronic liver disease (CLD), congestive cardiac failure (CCF), acute coronary syndrome (ACS), chronic renal failure (CRF), chronic obstructive pulmonary disease (COPD), deep vein thrombosis (DVT), and PE alone were compared among all four groups of anticoagulation discharge with reference to the length of hospital stay, as shown in Table I. Hyperthrombophilic state $(p<0.001)$, deep vein thrombosis $(p<0.012)$, COPD $(p<0.001)$, and CKD $(p<0.028)$ were found to be statistically significant for prolonging the length of stay in hospital among the groups discharged on anticoagulants, as shown in Table I.

\section{Discharged anticoagulants and length of hospital stay}

A significant overall statistical association $(p<0.001)$ was found between duration of stay in hospital and various anticoagulants on discharge such as rivaroxaban, warfarin sodium, enoxaparin with warfarin, and enoxaparin alone. Patients who were discharged on anticoagulation with warfarin had significantly longer LOS compared to those sent home on rivaroxaban ( 7.65 vs. 5.21 days, $p<0.001$; Table II). Patients who were discharged exclusively on enoxaparin (3.30 days), or enoxaparin and warfarin (4.26 days), when compared for length of stay with rivaroxaban, also showed statistical significance $(p<0.0001)$.

\section{Outcome measures}

\section{Bleeding complications}

Bleeding complications were assessed and compared as an outcome through univariate analysis among the discharged anticoagulant patients. Major bleeding complications appeared in five (10.9\%) patients on rivaroxaban, seven (10.1\%) with warfarin, two (6.7\%) on enoxaparin plus warfarin therapy, and in $0(0 \%)$ enoxaparin-taking patients. Mild 
Table I. Length of Stay among patients discharged on various anticoagulants (clinical risk factors)

\begin{tabular}{|c|c|c|c|c|c|c|c|c|c|}
\hline Groups & \multicolumn{2}{|c|}{ Warfarin (69) } & \multicolumn{2}{|c|}{$\begin{array}{c}\text { Enoxaparin } \\
\text { and warfarin (30) }\end{array}$} & \multicolumn{2}{|c|}{ Rivaroxaban (46) } & \multicolumn{2}{|c|}{ Enoxaparin (16) } & $P$-value \\
\hline Age (years) & \multicolumn{2}{|c|}{$55.50 \pm 14.68$} & \multicolumn{2}{|c|}{$59.69 \pm 11.54$} & \multicolumn{2}{|c|}{$60.02 \pm 10.49$} & \multicolumn{2}{|c|}{$56.37 \pm 13.46$} & \\
\hline Male & 27 & 23 & $50.0 \%$ & $39.1 \%$ & 13 & $43.3 \%$ & 8 & $50.0 \%$ & 0.665 \\
\hline Hormones & 1 & 1 & $2.2 \%$ & $1.4 \%$ & 3 & $10.0 \%$ & 1 & $6.2 \%$ & 0.181 \\
\hline Immobilization & 3 & 5 & $10.9 \%$ & $4.3 \%$ & 6 & $20.0 \%$ & 2 & $12.5 \%$ & 0.113 \\
\hline Hyper thrombophilia & 4 & 6 & $13.0 \%$ & $5.8 \%$ & 9 & $30.0 \%$ & 11 & $68.8 \%$ & $<0.001$ \\
\hline Malignancy & 8 & 10 & $21.7 \%$ & $11.6 \%$ & 9 & $30.0 \%$ & 4 & $25.0 \%$ & 0.148 \\
\hline Surgery & 5 & 3 & $6.5 \%$ & $7.2 \%$ & 2 & $6.7 \%$ & 2 & $12.5 \%$ & 0.878 \\
\hline Post traumatic & 1 & 4 & $8.7 \%$ & $1.4 \%$ & 1 & $3.3 \%$ & 2 & $12.5 \%$ & 0.154 \\
\hline Surgery & 5 & 3 & $6.5 \%$ & $7.2 \%$ & 2 & $6.7 \%$ & 2 & $12.5 \%$ & 0.878 \\
\hline Congestive heart failure & 4 & 3 & $6.5 \%$ & $5.8 \%$ & 6 & $20.0 \%$ & 1 & $6.2 \%$ & 0.114 \\
\hline Diabetes mellitus & 22 & 18 & $39.1 \%$ & $31.9 \%$ & 15 & $50.0 \%$ & 8 & $50.0 \%$ & 0.284 \\
\hline Chronic kidney disease & 7 & 6 & $13.0 \%$ & $10.1 \%$ & 10 & $33.3 \%$ & 2 & $12.5 \%$ & 0.028 \\
\hline $\begin{array}{l}\text { Chronic obstructive pulmonary } \\
\text { disease }\end{array}$ & 4 & 3 & $6.5 \%$ & $5.8 \%$ & 1 & $3.3 \%$ & 7 & $43.8 \%$ & $<0.001$ \\
\hline Chronic liver disease & 1 & 2 & $4.3 \%$ & $1.4 \%$ & 1 & $3.3 \%$ & 1 & $6.2 \%$ & 0.707 \\
\hline Pulmonary embolism alone & 1 & 2 & $4.3 \%$ & $1.4 \%$ & 2 & $6.7 \%$ & 2 & $12.5 \%$ & 0.227 \\
\hline Deep vein thrombosis & 64 & 44 & $95.7 \%$ & $92.8 \%$ & 30 & $100.0 \%$ & 12 & $75.0 \%$ & 0.012 \\
\hline Unknown & 31 & 20 & $43.5 \%$ & $44.9 \%$ & 13 & $43.3 \%$ & 4 & $25.0 \%$ & 0.533 \\
\hline
\end{tabular}

Table II. Correlation of outcomes with various anticoagulants on discharge

\begin{tabular}{|c|c|c|c|c|c|c|c|c|c|}
\hline Groups & \multicolumn{2}{|c|}{ Warfarin sodium } & \multicolumn{2}{|c|}{$\begin{array}{c}\text { Warfarin } \\
\text { with enoxaparin }\end{array}$} & \multicolumn{2}{|c|}{ Rivaroxaban } & \multicolumn{2}{|c|}{ Enoxaparin alone } & $P$-value \\
\hline Length of Stay (days) & \multirow{2}{*}{\multicolumn{2}{|c|}{$\begin{array}{l}7.65 \pm 1.29 \\
7.73(1.55)\end{array}$}} & \multirow{2}{*}{\multicolumn{2}{|c|}{$\begin{array}{l}4.26 \pm 1.01 \\
4.35(1.74)\end{array}$}} & \multirow{2}{*}{\multicolumn{2}{|c|}{$\begin{array}{l}5.21 \pm 1.20 \\
5.55(1.98)\end{array}$}} & \multirow{2}{*}{\multicolumn{2}{|c|}{$\begin{array}{l}3.30 \pm 1.40 \\
3.79(2.55)\end{array}$}} & \multirow[t]{2}{*}{$<0.001$} \\
\hline Median (inter-quartile range) & & & & & & & & & \\
\hline Readmission & 3 & $4.3 \%$ & 2 & $6.7 \%$ & 3 & $6.5 \%$ & 1 & $6.2 \%$ & 0.949 \\
\hline Bleeding manifestations & & & & & & & & & 0.802 \\
\hline Major & 7 & $10.1 \%$ & 2 & $6.7 \%$ & 5 & $10.9 \%$ & 0 & $0.0 \%$ & \\
\hline Mild & 3 & $4.3 \%$ & 1 & $3.3 \%$ & 2 & $4.3 \%$ & 0 & $0.0 \%$ & \\
\hline None & 59 & $85.5 \%$ & 27 & $90.0 \%$ & 39 & $84.8 \%$ & 16 & $100.0 \%$ & \\
\hline
\end{tabular}

bleeding was observed in two (4.3\%) rivaroxaban patients, three $(4.3 \%)$ on warfarin, one (3.3\%) on enoxaparin plus warfarin, and in none of the enoxaparin-taking patients. Bleeding outcome when compared among anticoagulant discharged groups was found to be statistically insignificant $(p<0.802)$ (see Table II).

\section{Readmissions}

Readmissions, as another outcome parameter among these groups, were observed to be three $(6.5 \%)$ on rivaroxaban, three $(4.3 \%)$ on warfarin, two $(6.7 \%)$ on enoxaparin plus warfarin, and one (6.2\%) on enoxaparin. When compared, it was statistically insignificant, with a $p$ value of 0.949 (see Table II).

\section{Duration of hospital stay (independent predictors)}

Various independent predictors such as age, gender, and conditions such as COPD, CCF, and CKD were assessed by multivariate analysis for Length of Stay among groups of patients discharged on various anticoagulants. Anticoagulants like enoxaparin, enoxaparin + warfarin, and warfarin alone with reference to rivaroxaban were also assessed as independent predictors for Length of Stay among groups of patients. Among all independent predictors for Length of Stay, only the discharged anticoagulants enoxaparin, enoxaparin + warfarin, and warfarin were found to be significant ( $p<0.001,<0.03$ and $<0.001$ ) (see Table III). 
Table III. Independent predictors of longer hospital stay among patients with different anticoagulants

\begin{tabular}{|c|c|c|c|c|c|c|}
\hline \multirow{2}{*}{ Predictors } & \multirow{2}{*}{$\begin{array}{l}\text { Estimate } \\
\text { of regression }\end{array}$} & \multirow{2}{*}{$\begin{array}{l}\text { Standard error } \\
\text { of regression }\end{array}$} & \multirow{2}{*}{$t$} & \multirow{2}{*}{$P$-value } & \multicolumn{2}{|c|}{$\begin{array}{l}\text { 95\% confidence interval } \\
\text { for B }\end{array}$} \\
\hline & & & & & Lower bound & Upper bound \\
\hline \multicolumn{7}{|l|}{ Demography } \\
\hline Age & -0.00064 & 0.008 & -0.082 & 0.934 & -0.016 & 0.015 \\
\hline Gender & -0.013 & 0.202 & -0.064 & 0.949 & -0.413 & 0.387 \\
\hline Anticoagulants & & & & & & \\
\hline Warfarin & 2.358 & 0.243 & 9.723 & $<0.001$ & 1.879 & 2.838 \\
\hline Enoxaparin and warfarin & -0.913 & 0.306 & -2.983 & 0.003 & -1.518 & -0.308 \\
\hline Enoxaparin & -1.809 & 0.419 & -4.312 & $<0.001$ & -2.638 & 0.980 \\
\hline \multicolumn{7}{|l|}{ Clinical risk factors } \\
\hline Chronic kidney disease & -0.399 & 0.292 & -1.367 & 0.174 & -0.975 & 0.178 \\
\hline $\begin{array}{l}\text { Chronic obstructive pulmonary } \\
\text { airway disease }\end{array}$ & -0.175 & 0.391 & -0.447 & 0.656 & -0.948 & 0.598 \\
\hline Congestive cardiac failure & -0.221 & 0.357 & -0.619 & 0.537 & -0.927 & 0.485 \\
\hline \multicolumn{7}{|l|}{ Causes of VTE } \\
\hline Hyper-thrombophilia & 0.054 & 0.305 & 0.177 & 0.860 & -0.549 & 0.657 \\
\hline Hormonal & 0.180 & 0.547 & 0.330 & 0.742 & -0.900 & 1.261 \\
\hline Malignancy & -0.452 & 0.261 & -1.732 & 0.085 & -0.967 & 0.064 \\
\hline Immobilization & 0.482 & 0.342 & 1.411 & 0.160 & -0.193 & 1.158 \\
\hline Surgery & -0.544 & 0.392 & -1.388 & 0.167 & -1.319 & 0.231 \\
\hline
\end{tabular}

\section{Discussion}

Our study compared the effect of various anticoagulation drugs on patients with VTE. The duration of hospital stay, bleeding, and readmissions were assessed as outcome parameters. Age and gender distribution were compared among anticoagulation groups (warfarin, rivaroxaban, warfarin and enoxaparin, and enoxaparin alone) and found to be statistically insignificant. However, an earlier study [17] has shown age to be statistically significant among anticoagulation groups. The majority of patients in our study were on warfarin, followed by rivaroxaban, enoxaparin + warfarin, and enoxaparin alone, and this is in accordance with an earlier study [17]. Warfarin is the most cost-effective drug among all groups, which explains its being the most used anticoagulant group. Among various clinical risk factors such as thrombophilia, CCF, COPD, and CKD, only hyperthrombophilic state, deep vein thrombosis, COPD and CKD were statistically significant for prolonging the length of stay in hospital among the discharged groups of anticoagulants.

Ruggles et al. [18] have shown heart failure, CKD and coronary artery disease to be significant clinical risk factors for prolonging hospital stay among patients with thromboembolism on various anticoagulants. A previous similar study [17] has also shown CKD, malignancy and hypercoagulable state as significant risk factors prolonging hospitalization in patients with thromboembolism on anticoagulants. An earlier study [18] among intensive care unit (ICU) patients on anticoagulants had shown length of stay for warfarin to be 3.0 days [95\% confidence interval $(\mathrm{Cl})$ 1.9-3.9; $p<0.001$ ] more than for patients on dabigatran, and 2.4 days longer $(95 \% \mathrm{Cl} 0.9-3.7 ; p=0.003)$ than for patients on rivaroxaban. However, that study showed no difference in hospital stay between rivaroxaban and dabigatran, although it differed from the current study as they also compared novel anticoagulants like dabigatran and rivaroxaban.

Saint et al. [19] compared duration of hospital stay among patients managed through novel oral anticoagulants (rivaroxaban) with warfarin and warfarin plus a parenteral agent for venous thromboembolism. They found a shorter duration of hospital stay ( 2.63 vs. 5.33 days; $p<0.05$ ) for rivaroxaban. Our current study showed slightly more major bleeding in patients discharged on warfarin, although overall comparison of bleeding among various groups of anticoagulants showed no significant difference. When bleeding as an outcome parameter for length of hospital stay was compared among groups, length of stay was shortest with 
rivaroxaban. Ruggles et al. [18] also assessed length of stay among patients having bleeding due to various anticoagulant groups, and showed shortest length of stay with dabigatran and rivaroxaban as compared to warfarin. An earlier study [17] reported similar results to our study. Readmission as an outcome parameter in our study did not differ among various groups of anticoagulants, which is similar to the earlier study [17]. Readmission in our study was lower than that reported in the EINSTEIN trial [7]. Rivaroxaban, warfarin, enoxaparin plus warfarin, and enoxaparin alone were found to be significant independent predictors of length of stay in our study. The earlier study found similar results [17].

\section{Conclusions}

In our study, various demographic features and clinical characteristics of patients with VTE have been analyzed for consecutive durations of hospital stay, while comparing the impact to the array of contemporary versus novel anticoagulant drugs.

It is noteworthy that patients who were discharged on anticoagulation with warfarin had significantly longer LOS compared to those sent home on rivaroxaban $(p<0.001)$. Bleeding outcome and readmissions when compared among anticoagulant discharged groups were found to be statistically insignificant: $p<0.802$, and $p<0.949$ respectively. Among all independent predictors of length of stay, only discharged anticoagulants enoxaparin, enoxaparin + warfarin, and warfarin, were found to be significant $(p<0.001, p<0.03$ and $p<0.001$, respectively).

Therefore, our study concludes that the preference of novel anticoagulants has an observable impact on the length of hospital stay in patients with acute venous thromboembolism.

\section{Authors' contributions}

IHN and AT: design of study; SNZR: statistical analysis; MR, RZG and SKF: manuscript writing, data collection; AT: final approval

\section{Conflicts of interest \\ None.}

\section{Financial support \\ None.}

\section{Ethics}

The work described in this article has been carried out in accordance with The Code of Ethics of the World Medical Association (Declaration of Helsinki) for experiments involving humans; EU Directive 2010/63/EU for animal experiments; Uniform Requirements for manuscripts submitted to biomedical journals.

\section{References}

1. Oger $E$. Incidence of venous thromboembolism: a community-based study in Western France. Thromb Haemost. 2017; 83(05): 657-660, doi: 10.1055/s-0037-1613887.

2. Spencer FA, Emery C, Lessard D, et al. The Worcester Venous Thromboembolism study: a population-based study of the clinical epidemiology of venous thromboembolism. J Gen Intern Med. 2006; 21(7): 722-727, doi: 10.1111/j.1525-1497.2006.00458.x, indexed in Pubmed: 16808773.

3. Heit J, Cohen A, Anderson F, et al. Estimated annual number of incident and recurrent, non-fatal and fatal venous thromboembolism (VTE) events in the US. Blood. 2005; 106(11): 910-910, doi: 10.1182/blood.v106.11.910.910.

4. Goldhaber SZ, Visani L, De Rosa M. Acute pulmonary embolism: clinical outcomes in the International Cooperative Pulmonary Embolism Registry (ICOPER). Lancet. 1999; 353(9162): 1386-1389, doi: 10.1016/s0140-6736(98)07534-5, indexed in Pubmed: 10227218.

5. Kearon C, Akl EA, Comerota AJ, et al. Antithrombotic therapy for VTE disease: antithrombotic therapy and prevention of thrombosis, 9th ed: American College of Chest Physicians Evidence-Based Clinical Practice Guidelines. Chest. 2012; 141(2 Suppl): e419S-e496S, doi: 10.1378/chest.11-2301, indexed in Pubmed: 22315268.

6. Eriksson Bl, Quinlan DJ, Eikelboom JW. Novel oral factor Xa and thrombin inhibitors in the management of thromboembolism. Annu Rev Med. 2011; 62: 41-57, doi: 10.1146/annurev-med-062209-095159, indexed in Pubmed: 21226611.

7. Ramacciotti E, Agati LB, Calderaro D, et al. EINSTEIN Investigators, EINSTEIN-PE Investigators, EINSTEIN Investigators. Oral rivaroxaban for symptomatic venous thromboembolism. N Engl J Med. 2010; 363(26): 2499-2510, doi: 10.1056/NEJMoa1007903, indexed in Pubmed: 21128814.

8. Büller HR, Prins MH, Lensin AWA, et al. EINSTEIN-PE Investigators. Oral rivaroxaban for the treatment of symptomatic pulmonary embolism. N Engl J Med. 2012; 366(14): 1287-1297, doi: 10.1056/ NEJMoa1113572, indexed in Pubmed: 22449293.

9. Prins MH, Lensing AWa, Bauersachs R, et al. EINSTEIN Investigators. Oral rivaroxaban versus standard therapy for the treatment of symptomatic venous thromboembolism: a pooled analysis of the EINSTEIN-DVT and PE randomized studies. Thromb J. 2013; 11(1): 21, doi: 10.1186/1477-9560-11-21, indexed in Pubmed: 24053656.

10. van Bellen B, Bamber L, Correa de Carvalho F, et al. Reduction in the length of stay with rivaroxaban as a single-drug regimen for the treatment of deep vein thrombosis and pulmonary embolism. Curr Med Res Opin. 2014; 30(5): 829-837, doi: 10.1185/03007995.2013.879439, indexed in Pubmed: 24432872.

11. Bookhart BK, Haskell L, Bamber L, et al. Length of stay and economic consequences with rivaroxaban vs enoxaparin/vitamin $\mathrm{K}$ antagonist in patients with DVT and PE: findings from the North American EINSTEIN clinical trial program. J Med Econ. 2014; 17(10): 691-695, doi: 10.3111/13696998.2014.946993, indexed in Pubmed: 25065536.

12. Kahler ZP, Beam DM, Kline JA. Cost of treating venous thromboembolism with heparin and warfarin versus home treatment with rivaroxaban. Acad Emerg Med. 2015; 22(7): 796-802, doi: 10.1111/ acem.12713, indexed in Pubmed: 26111453.

13. American College of Radiology. Practical guidelines and standards for performance of the peripheral venous ultrasound examination. Reston 2015. 
14. van Belle A, Büller HR, Huisman MV, et al. Effectiveness of managing suspected pulmonary embolism using an algorithm combining clinical probability, D-dimer testing, and computed tomography. JAMA. 2006; 295(2): 172-179, doi: 10.1001/jama.295.2.172, indexed in Pubmed: 16403929

15. Ghaye B, Remy J, Remy-Jardin M. Non-traumatic thoracic emergencies: CT diagnosis of acute pulmonary embolism: the first 10 years. Eur Radiol. 2002; 12(8): 1886-1905, doi: 10.1007/s00330-002 1506-z, indexed in Pubmed: 12136308.

16. Gulsun M, Goodman LR, Washington L, et al. CT for thromboembolic disease. Radiol Clin North Am. 2002; 40(4): 751-771, doi: 10.1016/s0033-8389(02)00018-0, indexed in Pubmed: 12171183.
17. Desai $A$, Desai $A$, Calixte $R$, et al. Comparing length of stay between patients taking rivaroxaban and conventional anticoagulants for treatment of venous thromboembolism. Lung. 2016; 194(4): 605-611, doi: 10.1007/s00408-016-9898-8, indexed in Pubmed: 27192990.

18. Ruggles DR, Freyman RL, Oxenham AJ. Influence of musical training on understanding voiced and whispered speech in noise. PLoS One. 2014; 9(1): e86980, doi: 10.1371/journal.pone.0086980, indexed in Pubmed: 24489819.

19. Saint CA, Castelli MR, Crannage AJ, et al. Comparison of hospital length of stay in patients treated with non-vitamin $\mathrm{K}$ oral anticoagulants or parenteral agents plus warfarin for venous thromboembolism. SAGE Open Med. 2017; 5: 2050312117719628, doi: 10.1177/2050312117719628, indexed in Pubmed: 28781876. 